\title{
Interacción suelo-estructura en edificios de gran altura con subterráneos en Santiago, Chile
}

\author{
Soil-structure interaction of tall buildings with basement in Santiago, Chile
}

Fecha de entrega: 6 de diciembre 2018

Fecha de aceptación: 22 de marzo 2019

\section{Francisco J. Pinto-Vega ${ }^{1}$ y Christian Ledezma ${ }^{2}$}

${ }^{1}$ Departamento de Ingeniería Estructural y Geotécnica, Pontificia Universidad Católica de Chile, Avda. Vicuña Mackenna 4860, 7820436, Macul, Chile, fpinto1@uc.cl (Orcid: 0000-0003-4807-3391)

2 Departamento de Ingeniería Estructural y Geotécnica, Pontificia Universidad Católica de Chile, Avda. Vicuña Mackenna 4860, 7820436, Macul, Chile, ledezma@ing.puc.cl (Orcid: 0000-0003-3821-6264)

A la fecha, en el mundo existen cerca de 935 edificios con más de $200 \mathrm{~m}$ de altura. En Sudamérica, La Torre Costanera Center, Chile, con $300 \mathrm{~m}$ de altura, es un ejemplo de estas edificaciones. Las investigaciones y los códigos de diseño reconocen que en edificaciones de gran altura la interacción dinámica suelo-estructura (DSSI) es importante y puede modificar la respuesta sísmica respecto a estimaciones de modelos simplificados convencionalmente empleados en los análisis y códigos de diseño estructural. Las investigaciones muestran la necesidad de considerar apropiadamente variables como la profundidad de los subterráneos, el tipo de suelo alrededor de la subestructura, la altura de la edificación y las características de la demanda sísmica. En este artículo se evalúa el comportamiento sísmico de una edificación de gran altura con sótanos profundos considerando DSSI. El análisis emplea modelamiento directo bidimensional de elementos finitos no-lineales considerando las características de una edificación de gran altura con sótanos en el contexto chileno. Los principales resultados caracterizan los esfuerzos laterales símicos en los muros de retención de subterráneos y las diferencias entre la demanda sísmica de campo libre y a nivel del sello de fundación.

Palabras clave: edificios de gran altura, subterráneo, interacción dinámica suelo-estructura
There are currently 935 buildings with heights of $200 \mathrm{~m}$ or more around the world. The Costanera Center Tower, in Chile, with $300 \mathrm{~m}$ of height is an example of this type of construction in South America. Researchers and seismic design codes recognize that dynamic soil-structure interaction (DSSI) have significant effects on the seismic response of tall buildings. Previous studies have shown the need of considering the depth of basement, the retaining wall flexibility, the characteristics of the soil around the building base, the structure's height, and the characteristics of the input ground motion. This study examines the seismic response of a tall building with deep basement considering soil-structure interaction. The dynamic behaviour of this tall building is evaluated using non-linear finite element analysis. The main results discussed in this paper focus on the lateral seismic pressures on the building's basement walls, the differences between the free-field and the foundation-level ground motions, and the dynamic structural response in terms of the shear force distribution. These results highlight the importance of considering DSSI effects in the seismic response of tall buildings with deep basements, and help on improving our understanding of the seismic performance of such buildings.

Keywords: tall building, deep basement, dynamic soilstructure interaction

\section{Introducción}

Según el Council on Tall Buildings and Urban Habitat (www.ctbuh.org), a la fecha existen cerca de 935 edificaciones con más de $200 \mathrm{~m}$ de altura. Lo anterior es reflejo de un crecimiento exponencial en la construcción 
de edificaciones de gran altura (tall building, high-rise buildings o skyscrapers, términos empleados comúnmente en el habla inglesa). Poulos (2017) destaca que estas edificaciones representan importantes desafíos para la práctica ingenieril, pues muchos de los métodos de diseño y análisis tradicionales no son extrapolables más allá de la experiencia previa o definición bajo los cuales son establecidos. En este sentido, la seguridad sísmica de estas estructuras representa un tema de importancia como lo dejan entrever PEER (2017). Las recomendaciones de estos códigos y criterios operacionales condicionan a los edificios de gran altura a estar conformados en la mayoría de los casos por varios niveles de subterráneo, sistema de fundaciones especiales y elementos viga-columna conectados a un núcleo rígido como superestructura. Numerosos estudios, e.g. Stewart et al. (1999a, 1999b), Wolf (1989) y Kausel (2010), así como guías de diseño y desempeño sísmico, muestran que el comportamiento sísmico de estas edificaciones presenta importantes efectos de interacción dinámica suelo estructura (DSSI, por sus siglas en inglés). Fenómeno descrito bajo dos mecanismos que actúan simultáneamente: interacción cinemática e interacción inercial.

Avilés y Pérez-Rocha (1998) y Stewart (2000) a través de análisis numérico, considerando la profundidad del sistema de fundación, muestran que los efectos de la interacción cinemática en edificaciones son dependientes del grado de enterramiento de la fundación y de la dimensión en planta de la misma. A través de modelos simplificados de DSSI, Stewart y Tileylioglu (2007) mostraron que las diferencias entre el movimiento de campo libre y a nivel de la fundación son más notorias para edificaciones de gran altura con varios niveles de subterráneos embebidos en el terreno. Al mismo tiempo, identifican que la flexibilidad de los muros de retención de los sótanos controla los desplazamientos relativos entre la cota de fundación y la superficie, así como el comportamiento de esfuerzos internos en los elementos de la superestructura, fenómeno aún no considerado o simplificado en los procedimientos de análisis y diseño. En cuanto a la interacción inercial, se ha buscado establecer sus efectos en la respuesta estructural en función de parámetros adimensionales que consideran la geometría de la subestructura y la superestructura y, el volumen de suelo en interacción (Avilés y Pérez-Rocha, 1996; Veletsos y Meek, 1974; Kim y Stewart, 2003;
Pitilakis et al., 2008). En el caso de edificaciones de gran altura se ha identificado importante influencia de la DSSI en la respuesta de modos superiores, principalmente en la distribución de deriva (drift) y de corte de entrepiso (Naeim et al., 2008).

En el estudio de la DSSI mediante métodos de análisis numéricos o analíticos, destacan dos enfoques: el directo (Elementos Finitos o Elementos de contorno) y el de subestructura. A pesar de la existencia de estos métodos, la DSSI ha sido, en general, obviada o simplificada por razones de adecuación a la práctica ingenieril (Stewart y Tileylioglu, 2007; Naeim et al., 2008). Acorde a Stewart y Tileylioglu (2007), la mayoría del análisis estructural de edificaciones de gran altura se centra en considerar modelos numéricos que obvian o simplifican la DSSI al considerar la base de la estructura fija y el movimiento de campo libre como demanda. Naeim et al. (2008) evalúan los efectos de la DSSI en una edificación de 54 pisos con cuatro niveles de subterráneo, empleando modelos de DSSI con enfoque de subestructura. Entre los resultados destaca que el modelo empleado reproduce la respuesta sísmica de una edificación instrumentada para el sismo de Northridge de 1994, sin embargo, al emplear la tipología de modelos de análisis estructural de la práctica ingenieril no existe correspondencia con la respuesta sísmica. En este sentido, reconoce que los modelos numéricos empleados en la práctica ingenieril obvian parámetros como la presión sísmica del suelo en los subterráneos por efectos cinemáticos e inerciales de DSSI.

Adicionalmente, y enmarcado en el contexto anterior, los códigos de diseño y el estándar de la práctica de la ingeniería han dictado que los empujes sísmicos del suelo deberían incluirse en el diseño de estructuras de retención de tierra en regiones sísmicas. La práctica ingenieril y códigos de diseño, e.g. ASCE (2013) y FEMA 450 (2003), aceptan para la estimación de empujes sísmicos sobre muros de subterráneo el empleo de métodos clásicos, por ejemplo, el método de Mononobe-Okabe o método de estimación de empujes en muros de subterráneos de la norma chilena para el diseño de edificio NCh433 (2009). En la actualidad se han desarrollado ensayos en centrifuga, modelamiento numérico y modelos analíticos que intentan representar los empujes sísmicos en estructuras enterradas. Estos estudios han concluido de manera divergente que los métodos 
clásicos sobre-estiman y sub-estiman los empujes sísmicos trasmitidos a los muros (Ostadan, 2005; Al Atik y Sitar, 2010; Mikola et al., 2016; Vrettos et al., 2016). Al mismo tiempo, los estudios establecen la necesidad de identificar el comportamiento de las presiones sísmicas transmitidas por el suelo a estructuras de retención enterradas bajo determinadas condiciones de trabajo. Brandenberg et al. (2015) a través de modelos analíticos de muros de retención en subterráneos sin considerar la superestructura y considerando la interacción cinemática de la DSSI, demostraron que los desplazamientos diferenciales suelomuro son función de la profundidad del subterráneo, cuando los sótanos son relativamente altos con respecto a la longitud de onda sísmica que viaja por el suelo. Al mismo tiempo, Brandenberg et al. (2015) proponen un procedimiento para la estimación de las presiones sísmicas del suelo, adoptado por PEER (2017), como un método alternativo a los métodos clásicos para el diseño de subterráneos. No obstante, Brandenberg et al. (2015) reconocen la necesidad de estudios experimentales y simulaciones numéricas para validar el procedimiento para un rango de frecuencias de movimiento y configuraciones de muro, así como evaluar la contribución relativa de los efectos inerciales y formular recomendaciones detalladas para el diseño.

Bajo el contexto anterior, la presente investigación evalúa el comportamiento sísmico de edificaciones de gran altura dentro del contexto chileno, considerando los efectos DSSI. El análisis emplea modelamiento bidimensional de elementos finitos no-lineales considerando enfoque directo y enfoque de subestructura. Los resultados muestran importantes diferencias de los esfuerzos laterales símicos en los muros de retención de subterráneos, con y sin la presencia de la estructura. Al mismo tiempo, son señaladas las diferencias entre la demanda sísmica de campo libre y a nivel del sello de fundación, las cuales concuerdan con resultados de investigaciones previas. Estas diferencias muestran la importancia de considerar los efectos de la DSSI en la evaluación del desempeño sísmico de edificaciones de gran altura, tanto a nivel de la subestructura como de la superestructura. Es importante señalar que los presentes resultados forman parte de un proyecto de investigación más general que está actualmente en desarrollo.
Metodología

Caso de estudio

El alcance del presente estudio es considerar la tipología de las edificaciones chilenas, apegado a los criterios normativos de los códigos de diseño chileno y código normativos internacionales que abordan las edificaciones en consideración. En el contexto chileno dos tipologías de edificaciones de gran altura son comúnmente empleadas: 1) edificaciones con planta libre y sistema resistente a fuerza lateral conformado por núcleo con muros de cortante de hormigón armado y pórtico de viga-columna perimetral, 2) edificaciones con sistema de muros de cortante de hormigón armado longitudinal y transversal, dispuesto en forma de espina de pescado.

Enmarcado en el contexto anterior, es analizado un modelo simplificado de un edificio con planta libre y sistema de núcleo con muros de hormigón armado localizado hipotéticamente en la ciudad de Santiago, Chile. Las dimensiones y características de los elementos estructurales siguen el código chileno para diseño de edificaciones sismo-resistente NCh433 (2009) y la ACI318 (2005). La edificación tiene 55 pisos (altura cercana a los $200 \mathrm{~m}$ ) y 7 subterráneos (profundidad aproximada de 21 $\mathrm{m})$, simétrica en planta. El sistema resistente a fuerzas laterales corresponde a un sistema de marco columna-viga conectado a un núcleo de concreto armado. El sistema de fundación consiste en losas y zapatas infinitamente rígidas con espesor de $3.0 \mathrm{~m}$. Las Figuras 1a, 1b y 1c muestran una vista en elevación, corte en planta del subterráneo y corte en planta de la superestructura, respectivamente.

\section{Caracterización geotécnica}

Según el alcance de la presente investigación, la edificación es considerada emplazada sobre el perfil típico de depósito fluvial de la ciudad de Santiago, conocido como grava de Santiago. Este depósito se caracteriza por estar compuesto de grava arenosa con 3\% de contenido de finos, índice plástico entre 5 y $20 \%$ y tamaño de las partículas superior a $20 \mathrm{~cm}$ (Rodríguez-Roa, 2000). El perfil está conformado por una capa superior de 1.5 a $3.0 \mathrm{~m}$ de arcilla de baja plasticidad de media a alta consistencia, posteriormente se conforma una capa de grava que contiene limos de baja plasticidad. Esta capa alcanza una profundidad de 5 a $7 \mathrm{~m}$, con una cohesión de $15 \mathrm{kPa}$ y un ángulo de 
a)

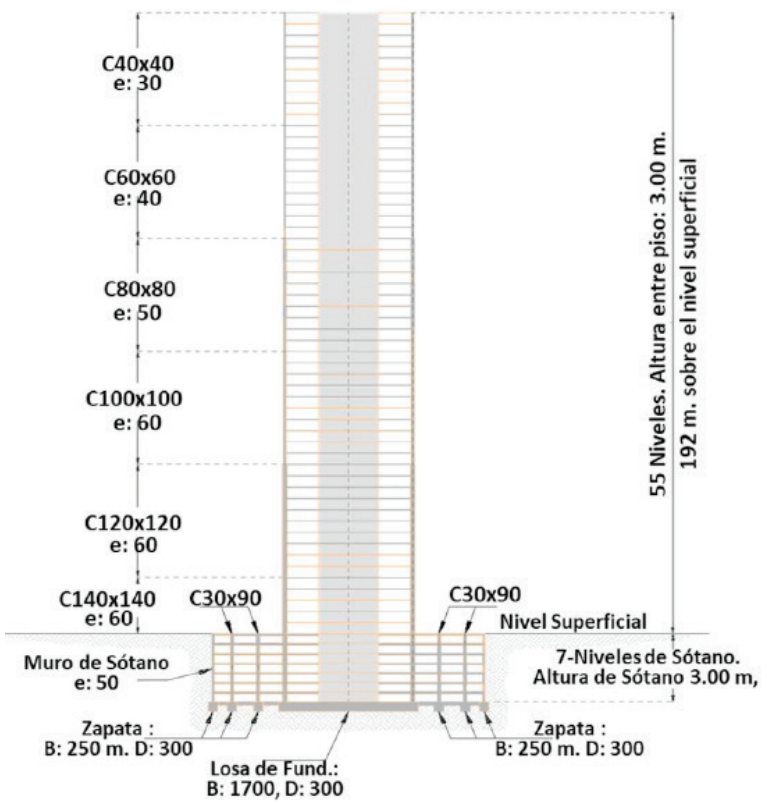

b)

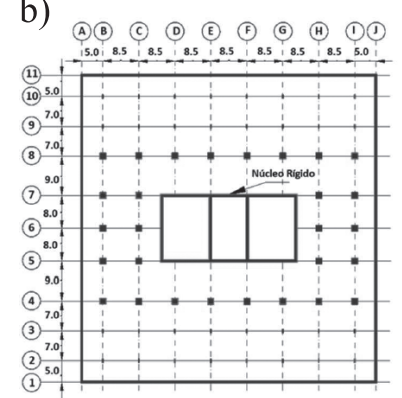

c)

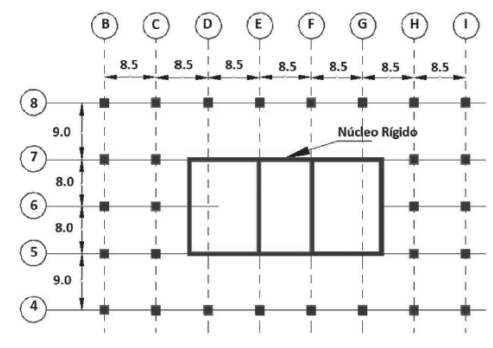

Figura 1: Edificacion de gran altura (caso de estudio). a) Perfil por el eje E, dimensiones en $\mathrm{cm}$, b) planta subterráneo S1-S7, dimensiones en $\mathrm{m}$ y c) planta de superestructura P1-P55, dimensiones en $\mathrm{m}$

fricción de $45^{\circ}$, este espesor de suelo es conocido como la segunda depositación del río Mapocho. Subyaciendo a la segunda depositación del río Mapocho, se tiene la primera depositación del río Mapocho. Este espesor de suelo presenta las mismas características granulométricas que la segunda depositación, pero resulta ser más densa, con parámetros geotécnicos de, aproximadamente, $25 \mathrm{kPa}$ de cohesión y $45^{\circ}$ de ángulo de fricción, e.g. Rodríguez-Roa (2000) y Ortigosa et al. (1982). La definición de parámetros elastoplásticos del suelo son abordados a través de parámetros representativos para el perfil de suelo de gravas de Santiago, e.g. Rodríguez-Roa (2000) y Salas (2018).

\section{Definición de la demanda sísmica}

El estudio realizado considera de manera explícita los efectos de sitio; por lo tanto, registros sísmicos de estaciones en roca son adecuados para el análisis. En este sentido, el registro sísmico de la estación Cerro el Roble del sismo de Maule de 2010 es empleado en el presente estudio (ver Figura 2). El registro presenta una aceleración máxima de $1.8 \mathrm{~m} / \mathrm{s}^{2}$ e Intensidad de Arias $1.5 \mathrm{~m} / \mathrm{s}$. Debido a las condiciones de medición en afloramiento rocoso (outcropping bedrock) del registro, fue realizado un proceso de deconvolución para aplicar el sismo en la base del modelo.

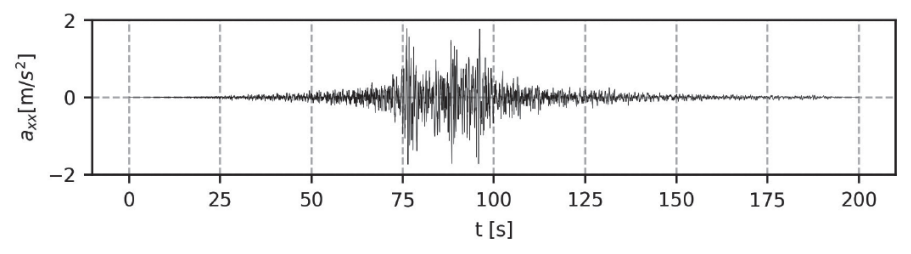

Figura 2: Componente N-S sismo de Maule 2010, estación Cerro El Roble

\section{Modelo de elementos finitos}

El comportamiento dinámico de la edificación en estudio está controlado, en gran medida, por el núcleo de hormigón armado del edificio a lo largo de su componente de translación lateral en la dirección más débil de la estructura, definido por un periodo predominante $T=6.2 \mathrm{~s}$, aproximadamente. Los modelos bidimensionales de elementos finitos se consideran, en una primera instancia, suficientemente precisos para representar el comportamiento dinámico bajo la acción de una demanda sísmica. A partir de lo señalado anteriormente, son desarrollados (1) un modelo directo suelo-fundación-estructura a través de la herramienta computacional Plaxis2D (Brinkgreve et al., 2018) y, (2) un modelo de subestructura con el suelo alrededor del subterráneo representado por curvas $p-y$ en el programa SAP2000 (Wilson y Habibullah, 1997). Las condiciones (1) y (2) requirieron estudiar la sensibilidad de los empujes sísmicos alrededor de las paredes de retención de tierra de los sótanos sin y con superestructura. Las Figuras 3a y 3 b muestran los modelos de elementos finitos desarrollados. No se muestra el modelo de base rígida por ser análogo al modelo directo de elemento finito, pero considera la estructura empotrada a nivel superficial.

Los modelos de elementos finitos a través del software Plaxis2D (Brinkgreve et al., 2018) consisten de dos subdominios. El primer subdominio está conformado por la superestructura, este subdominio es desarrollado a través de elementos plate para representar vigas, columnas y muros de retención en el sótano y, elementos solid para 


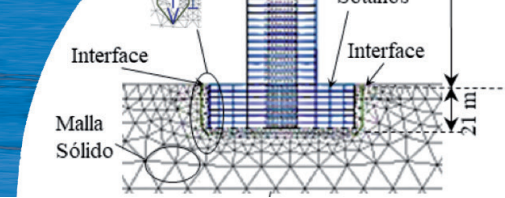

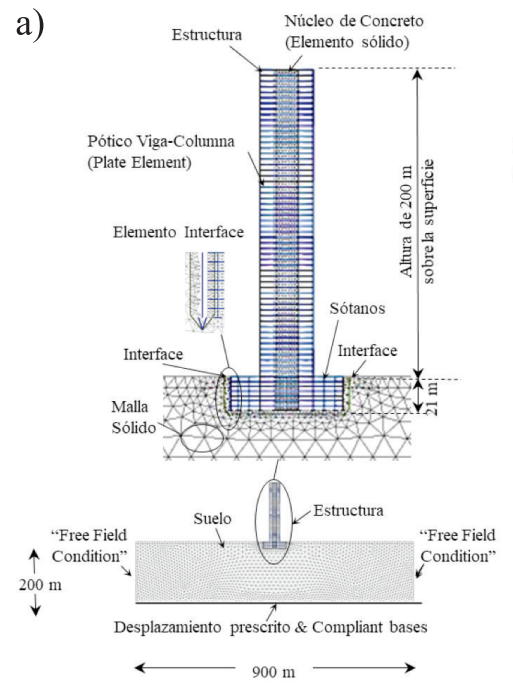

Figura 3: a) Modelo directo de elemento finito de DSSI y b) modelo de subestructura de DSSI

representar los muros del núcleo de la edificación y la cimentación de la subestructura. El segundo subdominio lo constituye el semi-espacio suelo o roca (dominio no finito), este segundo subdominio está compuesto por una discretización a través de elementos sólidos e incorporación de condiciones de borde artificiales para representar la condición semi-infinita del suelo. Ambos dominios, en el caso del modelo directo de elementos finitos, están en contacto a través de elementos interface entre los muros de retención y el suelo. La demanda sísmica es introducida en la base del modelo de elementos finitos. Los componentes de la superestructura son considerados elásticos, la fundación es considera elástica infinitamente rígida, el suelo es considerado no-lineal de tipo endurecimiento por deformación con pequeñas deformaciones (Hardening Soil Model with small strain, HS-Small) y, los elementos de interface responden a una condición por fricción de Mohr-Coulomb.

El modelo constitutivo usado para el suelo permite considerar la relación no lineal esfuerzo-deformación del suelo. Al mismo tiempo, tiene la ventaja durante el análisis dinámico de integrar la rigidez cortante a muy pequeñas deformaciones $\mathrm{y}$, considerar la degradación no lineal dependiendo de la amplitud de la deformación cortante experimentada por el suelo durante la carga dinámica. Los parámetros del modelo de comportamiento asumido para el suelo son abordados según Salas (2018), estos parámetros fueron definidos a partir de mediciones en campo con inclinómetro, modelamiento numérico y ensayos de laboratorio. Los parámetros empleados son resumidos en la Tabla 1.
Tabla 1: Parámetros del modelo constitutivo HS-Small

\begin{tabular}{|c|c|c|}
\hline Parámetro & $\begin{array}{c}\text { Primera } \\
\text { depositación }\end{array}$ & $\begin{array}{c}\text { Segunda } \\
\text { depositación }\end{array}$ \\
\hline Peso unitario $\gamma, \mathrm{kN} / \mathrm{m}^{3}$ & 23.5 & 23.5 \\
\hline Cohesión $c^{\prime}, \mathrm{kN} / \mathrm{m}^{2}$ & 35.0 & 15.0 \\
\hline Ángulo de fricción efectivo $\phi,^{\circ}$ & 45.0 & 45.0 \\
\hline Dilatancia, $\psi^{\circ}$ & 15.0 & 10.0 \\
\hline Grado de nolinealidad $m$ & 0.51 & 0.55 \\
\hline Rigidez secante $E_{50}{ }^{\text {ref }}, \mathrm{MN} / \mathrm{m}^{2}$ & 275 & 80 \\
\hline $\begin{array}{l}\text { Rigidez tangencial } E_{\text {oed }} \text { ref } \\
\mathrm{MN} / \mathrm{m}^{2}\end{array}$ & 190 & 130 \\
\hline $\begin{array}{l}\text { Rigidez carga/descarga } E_{\text {ur }} \text { ref } \\
\mathrm{MN} / \mathrm{m}^{2}\end{array}$ & 900 & 350 \\
\hline $\begin{array}{l}\text { Módulo cortante en pequeñas } \\
\text { deformaciones } G_{0}^{\text {ref }}, \mathrm{MN} / \mathrm{m}^{2}\end{array}$ & 750 & 400 \\
\hline $\begin{array}{l}\text { Deformación cortante al } 70 \% \\
\text { de } G_{\max }, \gamma_{0.7}\end{array}$ & 0.0011 & 0.0008 \\
\hline $\begin{array}{l}\text { Relación de Poisson en carga/ } \\
\text { descarga, } v_{u r}\end{array}$ & 0.2 & 0.3 \\
\hline $\begin{array}{l}\text { Coeficiente de empuje en } \\
\text { reposo, } K_{0}^{\text {nc }}\end{array}$ & 0.43 & 0.65 \\
\hline
\end{tabular}

El modelo de enfoque directo es desarrollado considerando dos condiciones de análisis, análisis estático y análisis dinámico. Las propiedades elásticas de los elementos estructurales para el análisis estático corresponden con la Tabla 2, propiedades como inercia y área de sección transversal, fueron estimadas directamente de la geometría de cada elemento. Estas propiedades geométricas fueron escaladas para ser consideradas por ancho tributario, siguiendo el espaciamiento entre ejes perpendiculares a la dirección más débil de la estructura. Los parámetros elásticos definidos para condición estática permiten garantizar la rigidez axial y a flexión compatible con los niveles de deformación esperados.

Tabla 2: Parámetros elásticos de elementos estructurales en análisis estático

\begin{tabular}{|l|c|c|c|c|}
\hline Parámetros & Viga & Columna & Núcleo & Fundación \\
\hline $\begin{array}{l}\text { Módulo elástico E, } \\
\text { GPa }\end{array}$ & 25 & 25 & 25 & 25 \\
\hline $\begin{array}{l}\text { Relación de } \\
\text { Poisson } v\end{array}$ & 0.20 & 0.20 & 0.15 & 0.20 \\
\hline $\begin{array}{l}\text { Peso unitario } \gamma, \\
\mathrm{kN} / \mathrm{m}^{3}\end{array}$ & 25 & 25 & 25 & 25 \\
\hline
\end{tabular}


Debido a limitaciones del programa de elementos finitos empleado que considera análisis bidimensional en deformaciones planas, un segundo conjunto de parámetros elásticos fue empleado para los elementos estructurales durante el análisis dinámico (ver Tabla 3), lo anterior permite alcanzar la frecuencia de vibración fundamental de la estructura considerando un modelo de base rígida o en roca. Por lo tanto, los nuevos parámetros garantizan que la estructura con interacción suelo-estructura conserve las características de vibraciones de la misma. La frecuencia fundamental objetivo corresponde con la determinada para la edificación durante el proceso de diseño de esta.

Tabla 3: Parámetros elásticos de elementos estructurales en análisis dinámico

\begin{tabular}{|l|c|c|c|c|}
\hline Parámetros & Viga & Columna & Núcleo & Fundación \\
\hline $\begin{array}{l}\text { Módulo elástico } \\
E, \mathrm{GPa}\end{array}$ & 25 & 5 & 5 & 25 \\
\hline $\begin{array}{l}\text { Relación de } \\
\text { Poisson } v\end{array}$ & 0.20 & 0.20 & 0.15 & 0.20 \\
\hline $\begin{array}{l}\text { Peso unitario } \gamma, \\
\mathrm{kN} / \mathrm{m}^{3}\end{array}$ & 25 & 25 & 25 & 25 \\
\hline
\end{tabular}

El mallado de elementos finitos en el semi-espacio suelo considera elementos triangulares de 15 nodos. El tamaño del elemento fue establecido garantizando al menos 8 elementos por longitud de onda, asociada a la frecuencia máxima con mayor contenido de energía del movimiento sísmico. La máxima frecuencia del movimiento sísmico fue $10 \mathrm{~Hz}$ y la mínima velocidad de onda de corte considerado en el modelo es $500 \mathrm{~m} / \mathrm{s}$; por lo tanto, el máximo tamaño del elemento empleado corresponde con $6.0 \mathrm{~m}$.

El modelo de subestructura a través del software SAP2000 consiste básicamente de un dominio (la superestructura) con condición de borde rígida en la base y elementos de reacción (springs) en los muros de contención del subterráneo. La superestructura está configurada a través de elementos beam para representar vigas, columnas y muros de retención en el sótano, elementos plate para representar los muros del núcleo de la edificación y elementos spring definidos con curvas $p$ - $y$ para representar el suelo en contacto con la subestructura. Las curvas $p-y$ son definidas según Reese et al. (1974) considerando arenas muy rígidas. Aunque el perfil de suelo está comprendido por un suelo gravoso, se considera la curva $p-y$ de arenas rígidas representativas de la condición de rigidez del suelo gravoso bajo las cargas dinámicas inducidas entre el contacto muro-suelo.

\section{Análisis y resultados}

Una primera evaluación de los efectos de la DSSI en la respuesta de parámetros sísmicos es realizada contrastando la demanda sísmica entregada a la estructura a nivel de la superficie y a nivel de la fundación. En la Figura 4, se compara la demanda sísmica a nivel de campo libre y a nivel de la fundación. La comparación es realizada en término del registro de aceleraciones y espectro de respuestas de aceleraciones. En primer lugar, al comparar el registro de aceleraciones de campo libre con la señal sísmica, se observa efectos de amplificación en el movimiento de traslación a nivel de la superficie, lo cual puede ser atribuido a condiciones de efecto de sitio. En segundo lugar, la Figura $4 \mathrm{~b}$ muestra diferencias entre el registro de aceleraciones de la señal en campo libre y a nivel de la fundación, estas diferencias corresponden con una menor amplitud de la aceleración medida a nivel de la fundación. La relación entre la amplitud espectral del movimiento sísmico en superficie $\left(P S A_{f g}\right)$ y la amplitud espectral del movimiento sísmico a nivel de la fundación $\left(P S A_{F I M}\right)$ es de 0.75 . Esta relación concuerda con lo señalado por Stewart y Tileylioglu (2007), atribuyendo esta diferencia a efectos cinemáticos e inerciales de la interacción dinámica sueloestructura y, relacionados principalmente a la flexibilidad del sistema suelo estructura y el desplazamiento relativo entre la estructura y el suelo alrededor de la estructura.

Con el objetivo de observar los efectos de la interacción dinámica suelo-estructura en los esfuerzos transmitidos en las paredes de retención del sótano, la Figura 5 muestra la variación con la profundidad del empuje estático más sísmico $\sigma_{\text {ae }}$ en el muro izquierdo del subterráneo sin considerar la estructura y considerando la estructura de modelos directos de elementos finitos. Al mismo tiempo, es presentada la media, media más o menos (+/-) desviación estándar estimada para las condiciones a compresión de los pasos del análisis tiempo historia realizado, la estimación del empuje estático más dinámico a través de M-O asumiendo una distribución triangular invertida (comúnmente empleado en la práctica ingenieril), la estimación del empuje de reposo aparente según la FHWA (Sabatini et al., 1999) más el empuje sísmico $\sigma_{\mathrm{s}}$ estimado según la NCh433 (2009) y, la distribución de presiones con 
a)

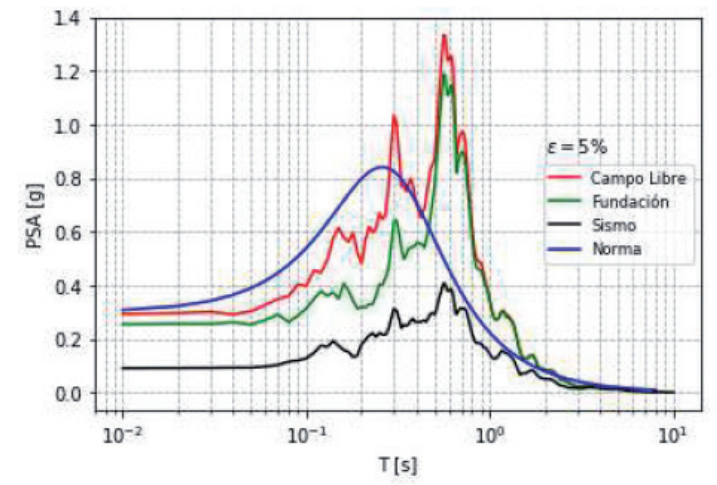

b)
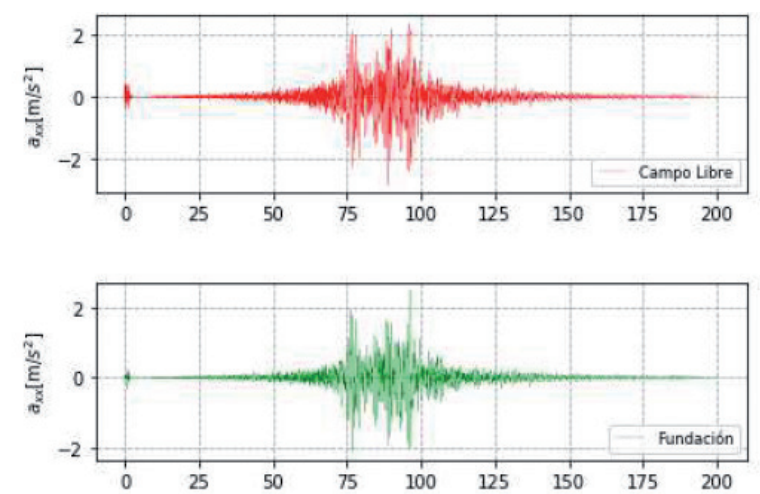

Figura 4: Comparación de la demanda sísmica de campo libre y a nivel de la fundación usando el sismo Cerro El Roble. a) Espectros de pseudo aceleraciones con estructura y b) aceleración versus tiempo.

la mayor resultante de fuerza transmitida al subterráneo $\sigma_{\text {aemax }}$. La Figura 5 muestra importantes contrastes entre el empuje transmitido a las paredes del subterráneo de modelos con y sin superestructura. La diferencia observada se hace más notable en los primeros $11 \mathrm{~m}$ de profundidad, afectando principalmente 4 niveles de la subestructura. Al mismo tiempo, como la literatura señala, en el caso de modelo sin estructura las presiones sísmicas transmitidas tienden a ser mucho menores que las estimadas con métodos clásicos. Caso contrario a lo señalado ocurre cuando se considera la superestructura donde los empujes en los primeros metros alcanzan la condición activa más sismo, en algunos casos sobrepasa esta condición. Sin embargo, observando la media y la media más desviación estándar de $\sigma_{\mathrm{ae}}$ y la condición $\sigma_{\mathrm{aemax}}$ en ambos casos (sin y con superestructura) se puede indicar que entre los $5 \mathrm{y}$ $6 \mathrm{~m}$ (aproximadamente) los empujes quedan envueltos por los métodos de diseños empleados en la práctica ingenieril. Lo anterior puede explicar el buen comportamiento de los muros de contención en los subterráneos de edificaciones de gran altura chilenas durante el terremoto de Maule del 2010.

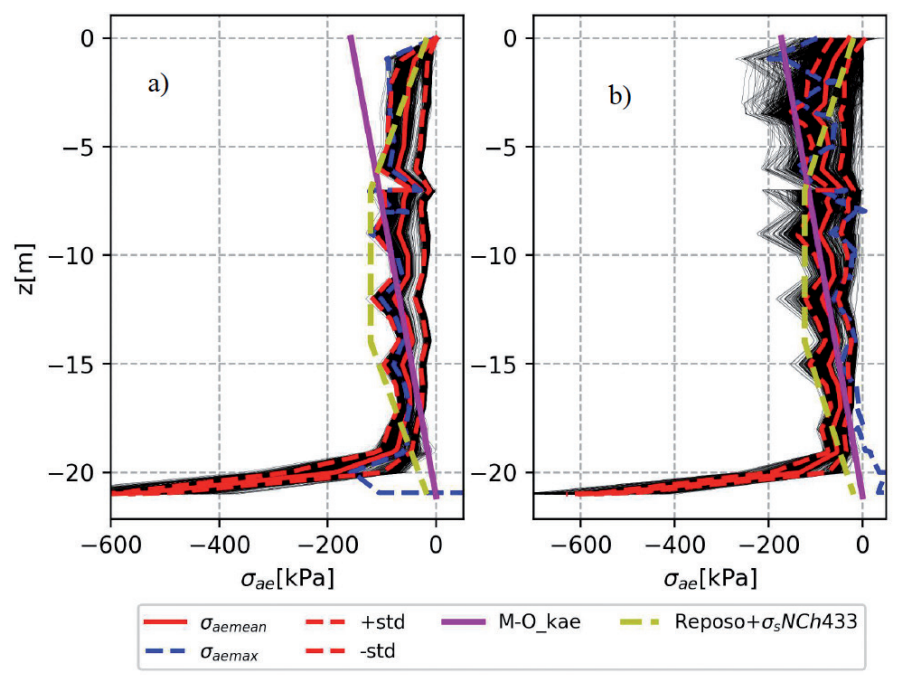

Figura 5: Empujes estáticos más sísmicos $\sigma_{\mathrm{ae}}$ transmitidos en las paredes de retención de los sótanos de modelos directos usando el registro del sismo Cerro El Roble, a) sin superestructura y b) con superestructura.

En el mismo contexto, se busca observar la contribución del empuje sísmico a la diferencia observada a través del modelo directo de DSSI con y sin estructura. En la Figura 6 se muestra la envolvente de la totalidad del análisis dinámico a través de modelos directos, la media de la variación con la profundidad de la componente dinámica en compresión del empuje sísmico sobre las paredes de retención del sótano de modelos directos y modelos de subestructura considerando simplificaciones con curvas $p-y \mathrm{y}$, la distribución de presiones sísmicas correspondiente a la mayor resultante de fuerza estática-dinámica transmitida al subterráneo $\Delta \sigma_{\text {emax }}$. Bajo el contexto anterior son presentados los resultados para modelos con y sin estructura. Al mismo tiempo, son incorporadas las presiones dinámicas sobre muros de retención estimadas a partir de métodos clásicos comúnmente empleado en la práctica ingenieril, como son el método de Mononobe-Okabe (MO_ke) y, el método de la norma NCh433. La Figura 6a muestra que las presiones sísmicas (media, $\Delta \sigma_{\text {emax }}$ y la totalidad de la presiones para el evento en consideración) sobre el subterráneo sigue la tendencia del empuje sísmico que se pueden estimar con el método clásico MO_ke; sin embargo se escapa de las estimaciones de empujes a partir de la norma NCh433. Por otro lado, la Figura $6 \mathrm{~b}$ señala que la presión sísmica aumenta considerablemente al incorporar la estructura. Al igual que lo anteriormente descrito, las presiones sísmicas para $\Delta \sigma_{\text {emax }}$ sigue la tendencia de las estimaciones a partir 
de MO_ke; sin embargo, la media de los resultados sigue la tendencia del método de estimación de presiones sísmica de la norma $\mathrm{NCh} 433$.

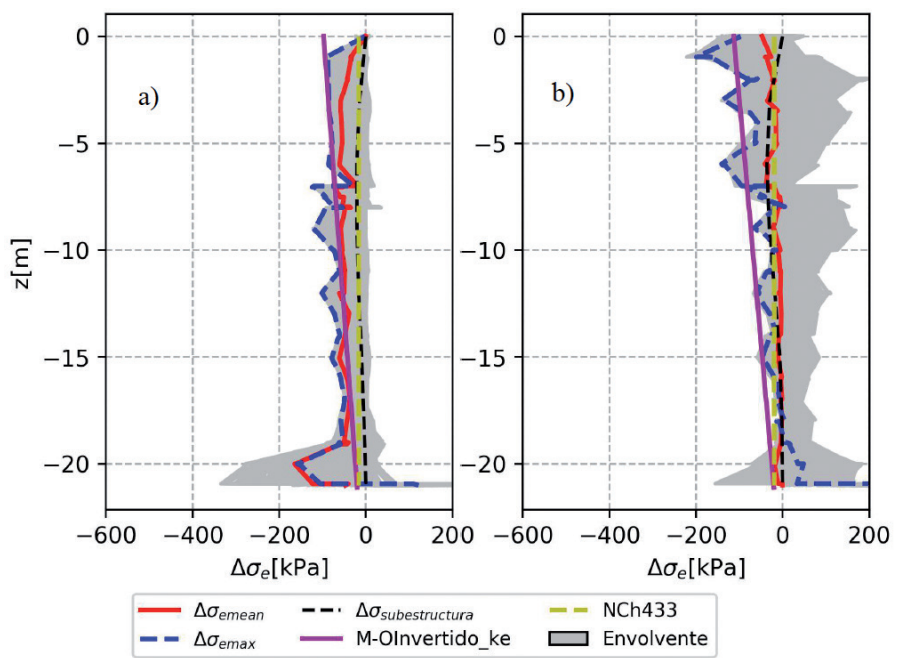

Figura 6: Empujes sísmicos $\Delta \sigma_{\mathrm{e}}$ transmitidos en los muros de los sótanos (usando el registro del sismo Cerro El Roble) de modelos directos y modelos de sub-estructura, a) sin superestructura y b) con superestructura.

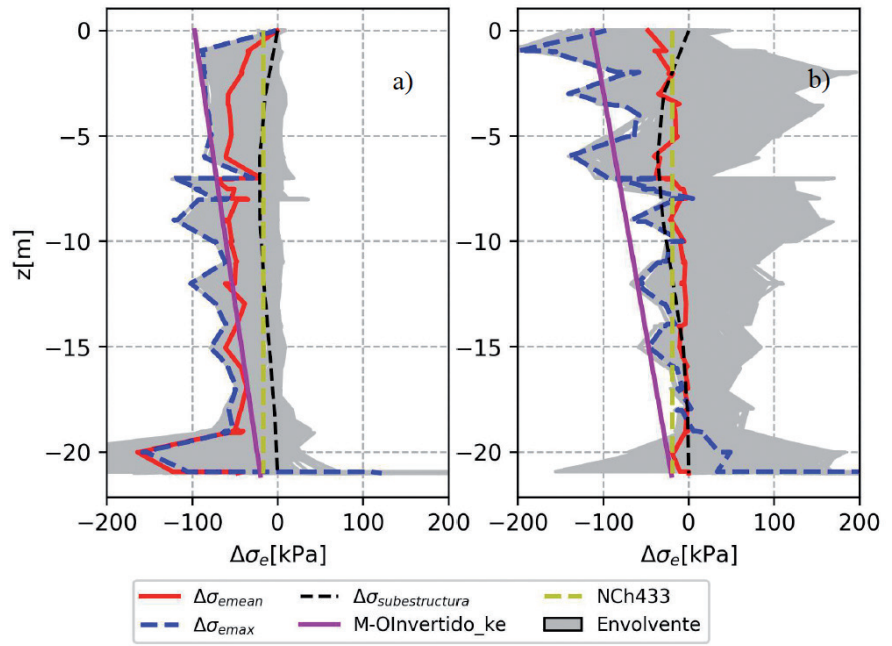

Figura 7: Empujes sísmicos $\Delta \sigma_{\mathrm{e}}$ transmitidos en los muros de los sótanos (usando el registro del sismo Cerro El Roble) de modelos directos y modelos de sub-estructura, a) sin superestructura y b) con superestructura.

Para una mayor claridad, la Figura 7 presenta una versión ampliada de la Figura 6. La Figura 7 resalta lo anteriormente expuesto, es decir que, para el caso de estudio, la incorporación de la estructura modifica (ampliamente) los empujes sísmicos que se generan a nivel de las paredes del subterráneo. Al mismo tiempo, se puede indicar que el empleo de diversas metodologías de estimación de empujes de tierras sobre muros no se adapta a la distribución y magnitud de empujes sísmicos transmitidos en las paredes de retención de tierra de los sótanos. Especial atención debe prestarse al efecto de descompresión del empuje sísmico transmitido a la estructura, este efecto ocurre a partir de los $7 \mathrm{~m}$ de profundidad (aproximadamente). Es importante señalar que la falta de similitud entre el modelo directo y el modelo de subestructura se puede asociar a que la asignación de las curvas $p-y$ no fue realizada a lo largo de la extensión de las paredes de retención del subterráneo, una mejor discretización puede arrojar una mejor semejanza entre las curvas. Sin embargo, existe suficiente concordancia en la tendencia observada.

\section{Conclusiones}

A partir de los resultados presentados se ve la necesidad de evaluar el comportamiento dinámico de edificaciones de gran altura con sótano considerando la interacción dinámica suelo-estructura. Importantes parámetros de respuesta sísmica de la edificación tanto a nivel de la subestructura, como de la superestructura y del suelo alrededor de la edificación se ven modificados, en contraposición a los análisis estructurales sísmicos que simplifican las condiciones de borde al estar completamente rígidas en la base. En primer lugar, la distribución de presiones sísmicas de tierra a nivel de los muros de retención del subterráneo, para el caso estudiado, no sigue la típica distribución de presiones sísmicas de los métodos clásicos empleado para el diseño de estos elementos geotécnicos. Al mismo tiempo, se observa que la incorporación de la estructura modifica el comportamiento de la distribución de presiones. Lo anterior sugiere, que efectos relativos a la dependencia del movimiento suelo-estructura son incorporados en la respuesta dinámica del suelo. Estos efectos según las evaluaciones realizadas pueden estar controlados por: la respuesta dinámica de la estructura, la flexibilidad de los muros de retención, y el contacto muro-suelo, entre otros aspectos. Segundo, el movimiento de campo libre y a nivel de la fundación son diferentes en términos de amplitud espectral y amplitud del tiempo-historia de aceleraciones. Finalmente, importantes efectos se observan al involucrar DSSI en la evaluación del comportamiento dinámico de edificaciones de gran altura. Los efectos observados 
sugieren la necesidad de realizar estudios adicionales que permitan una adecuada caracterización de estos a nivel de la subestructura.

\section{Agradecimentos}

Los autores agradecen el financiamiento de la beca Doctorado Nacional 2017 que ha permitido el desarrollo de la presente investigación.

\section{Referencias}

ACI-318 (2005). Building code requirements for structural concrete and commentary (318-05). Detroit, Michigan, USA

Al Atik, L. and Sitar, N. (2010). Seismic earth pressures on cantilever retaining structures. Journal of Geotechnical and Geoenvironmental Engineering 136(10), 1324-1333

ASCE (2013). Minimum design loads for buildings and other structures. Structural Engineering Institute SEI of the American Society of Civil Engineers ASCE, Reston, VA, USA

Avilés, J. and Pérez-Rocha, L.E. (1998). Effects of foundation embedment during building-soil interaction. Earthquake Engineering \& Structural Dynamics 27(12), 1523-1540

Avilés, J. and Pérez-Rocha, L.E. (1996). Evaluation of interaction effects on the system period and the system damping due to foundation embedment and layer depth. Soil Dynamics and Earthquake Engineering 15(1), 11-27

Brandenberg, S.J., Mylonakis, G. and Stewart, J.P. (2015). Kinematic framework for evaluating seismic earth pressures on retaining walls. Journal of Geotechnical and Geoenvironmental Engineering 141(7), 04015031

Brinkgreve, R.B.J., Kumarswamy, S., Swolfs, W.M. and Foria, F. (2018). Plaxis 2D Introductory: Manual General Information. Delft University of Technology and Plaxis bv, The Netherlands FEMA 450 (2003). NEHRP recommended provisions for seismic regulations for new buildings and other structures. National Earthquake Hazards Reduction Program NEHRP, Building Seismic Safety Council. Washington, D.C., USA

Kausel, E. (2010). Early history of soil-structure interaction. Soil Dynamics and Earthquake Engineering 30(9), 822-832

Kim, S. and Stewart, J.P. (2003). Kinematic soil-structure interaction from strong motion recordings. Journal of Geotechnical and Geoenvironmental Engineering 129(4), 323335
Mikola, R.G., Candia, G. and Sitar, N. (2016). Seismic earth pressures on retaining structures and basement walls in cohesionless soils. Journal of Geotechnical and Geoenvironmental Engineering 142(10), 04016047

Naeim, F., Tileylioglu, S., Alimoradi, A. and Stewart, J.P. (2008). Impact of foundation modeling on the accuracy of response history analysis of a tall building. Seminar on Utilization of Strong Motion Data, SMIP2008, 19-55

NCh433 (2009). Diseño sísmico de edificios. Instituto Nacional de Normalización INN, Santiago, Chile

Ortigosa, P., Musante, H. y Kort, I. (1982). Propiedades mecánicas de la grava de Santiago. Primer Congreso Chileno de Ingeniería Geotécnica, 442-454

Ostadan, F. (2005). Seismic soil pressure for building walls: An updated approach. Soil Dynamics and Earthquake Engineering 25(7-10), 785-793

PEER (2017). Guidelines for performance-based seismic design of tall buildings. Version 2.03. PEER Report 2017/06, Tall Building Initiative TBI, Pacific Earthquake Engineering Research Center, Berkeley, USA

Pitilakis, D., Dietz, M., Wood, D.M., Clouteau, D. and Modaressi, A. (2008). Numerical simulation of dynamic soilstructure interaction in shaking table testing. Soil Dynamics and Earthquake Engineering 28(6), 453-467

Poulos, H.G. (2017). Tall building foundation design. CRC Press Reese, L.C., Cox, W.R. and Koop, F.D. (1974). Analysis of laterally loaded piles in sand. Offshore Technology Conference, OTC 2080, Dallas, USA, 95-105

Rodríguez-Roa, F. (2000). Observed and calculated loadsettlement relationship in a sandy gravel. Canadian Geotechnical Journal 37(2), 333-342

Sabatini, P.J., Pass, D.G. and Bachus, R.C. (1999). Geotechnical engineering circular No. 4. Ground Anchors and Anchored Systems. FHWA-IF-99-015, Washington, DC, USA

Salas, F. (2018). Monitoring and dynamic analysis of a discontinuous pile-supported deep excavation in Santiago Gravel. MSc thesis, Pontificia Universidad Católica de Chile

Stewart, J.P. (2000). Variations between foundation-level and free-field earthquake ground motions. Earthquake Spectra 16(2), 511-532 
Stewart, J.P. and Tileylioglu, S. (2007). Input ground motions for tall buildings with subterranean levels. The Structural Design of Tall and Special Buildings 16(5), 543-557

Stewart, J.P., Fenves, G.L. and Seed, R.B. (1999a). Seismic soilstructure interaction in buildings. I: Analytical methods. Journal of Geotechnical and Geoenvironmental Engineering 125(1), 26-37

Stewart, J.P., Seed, R.B. and Fenves, G.L. (1999b). Seismic soilstructure interaction in buildings. II: Empirical findings. Journal of Geotechnical and Geoenvironmental Engineering 125(1), $38-48$

Veletsos, A.S. and Meek, J.W. (1974). Dynamic behaviour of building-foundation systems. Earthquake Engineering \& Structural Dynamics 3(2), 121-138
Vrettos, C., Beskos, D.E. and Triantafyllidis, T. (2016). Seismic pressures on rigid cantilever walls retaining elastic continuously non-homogeneous soil: An exact solution. Soil Dynamics and Earthquake Engineering 82, 142-153

Wilson, E. L. and Habibullah, A. (1997). SAP2000: integrated finite element analysis and design of structures. Computers and Structures, Berkeley, California, USA

Wolf, J.P. (1989). Soil-structure-interaction analysis in time domain. Nuclear Engineering and Design 111(3), 381-393 